\title{
Excitation of Radial Ion Motion in an rf-Only Multipole Ion Guide Immersed in a Strong Magnetic Field Gradient
}

\author{
Steven C. Beu, ${ }^{1}$ Christopher L. Hendrickson, ${ }^{2,3}$ Alan G. Marshall ${ }^{2,3}$ \\ ${ }^{1}$ S. C. Beu Consulting, 12449 Los Indios Trail, Austin, TX 78729, USA \\ ${ }^{2}$ Department of Chemistry and Biochemistry, Florida State University, 95 Chieftain Way, Tallahassee, FL 32306, USA \\ ${ }^{3}$ Ion Cyclotron Resonance Program, National High Magnetic Field Laboratory, Florida State University, 1800 East Paul Dirac \\ Drive, Tallahassee, FL 32310, USA
}

\begin{abstract}
Radiofrequency (rf) multipole ion guides are widely used to transfer ions through the strong magnetic field gradient between source and analyzer regions of external source Fourier transform ion cyclotron resonance mass spectrometers. Although ion transfer as determined solely by the electric field in a multipole ion guide has been thoroughly studied, transfer influenced by immersion in a strong magnetic field gradient has not been as well characterized. Recent work has indicated that the added magnetic field can have profound effects on ion transfer, ultimately resulting in loss of ions initially contained within the multipole. Those losses result from radial ejection of ions due to transient cyclotron resonance that occurs when ions traverse a region in which the magnetic field results in an effective cyclotron frequency equal to the multipole if drive frequency divided by the multipole order (multipole order is equal to one-half the number of poles). In this work, we describe the analytical basis for ion resonance in a rf multipole ion guide with superposed static magnetic field and compare with results of numerical trajectory simulations.
\end{abstract}

Key words: Ion cyclotron resonance, Fourier transform, FT-ICR, FTMS, Quadropole, Hexapole, Octopole

\section{Introduction}

$\mathrm{H}$ igh-pressure ionization techniques such as electrospray ionization (ESI), matrix-assisted laser desorption ionization (MALDI), and atmospheric pressure photoionization (APPI) are typically interfaced to FT-ICR MS with an

Electronic supplementary material The online version of this article (doi:10.1007/s13361-010-0057-9) contains supplementary material, which is available to authorized users.

Correspondence to: Alan G. Marshall; e-mail: marshall@magnet.fsu.edu, Christopher L. Hendrickson; e-mail: hendrick@magnet.fsu.edu external source, requiring ions to traverse a large magnetic field gradient during travel from the source to the analyzer [1]. To minimize the associated "magnetic mirror" effect [2] and optimize injection efficiency, ion optics are designed to accelerate and confine ions near the symmetry axis of the solenoidal magnetic field of a high-field superconducting magnet. Radiofrequency ( $\mathrm{rf}$ ) multipole ion guides have been among the most widely adopted ion optics for this purpose $[3,4]$. Although ion transfer as determined solely by electric fields in multipole ion guides has been thoroughly studied [5-8], transfer influenced by immersion in a strong magnetic field gradient has not been as well characterized [4, 9].

Several groups have described the motion of ions in a socalled combined trap in which both rf and magnetic fields serve to confine ions [10-16]. Those groups considered a quadrupole trap for which, in the absence of a magnetic 
field, the equations of motion in the two radial dimensions are separable and can be independently cast as Mathieu functions with well known solutions. Introduction of an axial magnetic field couples motion in the two radial dimensions; however, transformation to a coordinate frame rotating at one-half the cyclotron frequency decouples the equations for a 3D trap $[10,15]$. Contrary to claims in prior work [16], the lower symmetry of a 2D multipole field does not permit this transformation [Supplementary Material]; however, an approximate solution invoking a time-averaged electric field has been demonstrated [13].

Recent trajectory simulations have indicated that a magnetic field can have profound effects on ion kinetic energy and transfer in multipoles used for external source FT-ICR MS [17-20], ultimately resulting in loss of ions initially contained within the multipole $[17,18]$. Those losses result from radial ejection of ions due to transient cyclotron resonance that occurs when ions traverse a region where the prevailing magnetic field strength results in an effective cyclotron frequency equal to the multipole $\mathrm{rf}$ drive frequency divided by the multipole order (multipole order is equal to one-half the number of poles) (Figure 1). Experimental observation of ion loss consistent with this mechanism in a quadrupole injection FT-ICR MS has been

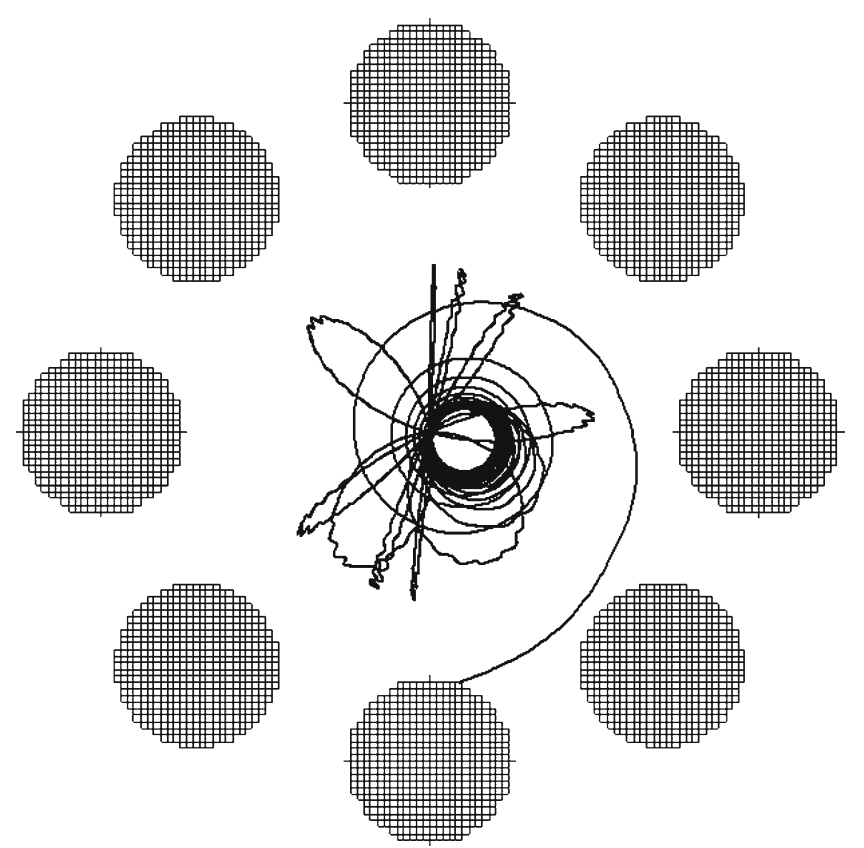

Figure 1. Radial trajectory of an ion ejected from a magneto-octopole. The ion initially oscillates in a plane containing the multipole axis. As the ion traverses a continuously increasing magnetic field, the plane of oscillation begins to precess around the axis and the trajectory assumes the shape of the rose curve. The ion ultimately encounters a magnetic field at which the ion cyclotron frequency equals the if drive frequency divided by the multipole order, and the trajectory assumes a spiral path resulting from continuous radial acceleration by the resonant electric field. The multipole if potential is $150 V_{p-p}$ at $1.5 \mathrm{MHz}$, and ion $\mathrm{m} / \mathrm{z}$ is 600 recently reported [21]. Here, we describe the analytical basis for ion resonance in a radiofrequency multipole ion guide with superposed static magnetic field (hereinafter referred to as a magnetomultipole) and compare with results of numerical trajectory simulations.

\section{Methods}

Computer simulations were performed with SIMION 3D ver. 7 (Idaho National Engineering and Environmental Laboratory, Idaho Falls, ID, USA) and SIMION ver. 8 (Scientific Instrument Services, Ringoes, NJ, USA) running on homebuilt 2.8 GHz AMD Athlon 64 X2 PCs with 2 GB ram. rf-Only quadrupole, hexapole, and octopole geometries with an inscribed radius of $2.38 \mathrm{~mm}$ and a length of $1.25 \mathrm{~m}$ were modeled with an array resolution of $0.0635 \mathrm{~mm}$. Leastsquares curve fitting and near-axis derivative approximations were used to derive an analytical description of the threedimensional magnetic field components of the $14.5 \mathrm{~T}$ magnet at the National High Magnetic Field Laboratory, and incorporated in the simulations by means of SIMION user programs. Injection efficiency was determined from analysis of 1000 collision-free ion trajectory simulations acquired for each specified mass-to-charge ratio, $\mathrm{m} / \mathrm{z}$, and the effects of variation in acceptance aperture with multipole geometry were avoided by use of trajectories that originate on the multipole axis and inset $5 \mathrm{~mm}$ from the entrance end of the multipole. Initial ion kinetic energy was $100 \mathrm{eV}$ and initial trajectory vectors were randomly directed between 0 to $13^{\circ}$ of the axis to yield a continuous distribution of radial kinetic energy over the range 0 to $5 \mathrm{eV}$. This range was sufficient to determine the radial kinetic energy confinement limit for all of the multipole conditions employed in this work. Deviations from these conditions for other simulation purposes are noted in the text.

\section{Theory}

\section{Ion Motion in the Magnetomultipole}

The motion of a non-relativistic charged particle in an electromagnetic field can be derived from the Lorentz equation of motion

$$
m \frac{d \boldsymbol{v}}{d t}=q \boldsymbol{E}+q \boldsymbol{v} \times \boldsymbol{B}
$$

in which $m, q$, and $\boldsymbol{v}$ are the ion mass, charge and velocity, and $\boldsymbol{E}$ and $\boldsymbol{B}$ are the electric and magnetic fields. The electric field within an rf-only multipole can be described in Cartesian coordinates (with the z-axis coincident with the multipole axis of symmetry) by

$$
\begin{aligned}
\boldsymbol{E}=\left\{E_{x}, E_{y}\right\}= & \frac{-V_{p-\mathrm{p}} \cos (\Omega t+\phi)}{2 r_{0}^{n}} \\
& \times\left\{\frac{d \operatorname{Re}\left[(x+i y)^{n}\right]}{d x}, \frac{d \operatorname{Re}\left[(x+i y)^{n}\right]}{d y}\right\}
\end{aligned}
$$


in which $V_{p \text {-p }}, \Omega$, and $\phi$ are the peak-to-peak rf amplitude, frequency and initial phase, $r_{\mathbf{0}}$ is the inscribed radius of the multipole and $n$ is the multipole order $[5,22]$. For the simplest case of a homogeneous magnetic field aligned with the symmetry axis of the multipole, $\boldsymbol{B}=(0,0, B)$, and ignoring the resulting uncoupled motion in the $\mathrm{z}$ dimension, substitution of this expression for the electric field into Equation 1 results in the following equations of motion

$$
\begin{aligned}
m \frac{d^{2} x}{d t^{2}}= & -\frac{q V_{p-\mathrm{p}} \cos (\Omega t+\phi)}{2 r_{0}^{n}}\left\{\frac{d \operatorname{Re}\left[(x+i y)^{n}\right]}{d x}\right\} \\
& +q B \times \frac{d y}{d t} \\
m \frac{d^{2} y}{d t^{2}}= & -\frac{q V_{p-\mathrm{p}} \cos (\Omega t+\phi)}{2 r_{0}^{n}}\left\{\frac{d \operatorname{Re}\left[(x+i y)^{n}\right]}{d y}\right\} \\
& -q B \times \frac{d x}{d t}
\end{aligned}
$$

Equations $3 \mathrm{a}$ and $3 \mathrm{~b}$ comprise a system of coupled nonlinear differential equations that does not have an exact analytical solution and it is typical to instead seek approximate solutions by use of nonlinear dynamics procedures [23]. Here we use simplifying assumptions based on results of modeling to develop analytical approximations for ion power absorption and resonant frequency shift in the magnetomultipole.

\section{Characteristic Ion Motion}

The magnetomultipole combines the magnetic radial confinement of the Penning or ICR trap with the electrodynamic radial confinement of an $\mathrm{rf}$ multipole ion guide, and the characteristic types of ion motion associated with each of those confinement mechanisms is described elsewhere [5, $24,25]$. We will briefly review only the radial ion motion here to provide a context for describing analogous motion in the magnetomultipole.

\section{Static Electromagnetic Ion Trap}

The principal radial motion observed in a magnetic trap is the cyclotron gyration caused by the magnetic force on an ion with a velocity component perpendicular (radial) to the magnetic field. In a Penning/ICR trap this effective magnetic confinement in the radial dimension is supplemented with an electrostatic field to confine motion in the direction parallel to the magnetic field (the axial dimension). For any electrode structure, the associated electrostatic field has a radial component in addition to the desired axial component. The resulting outward-directed radial force on the ion opposes the inward-directed magnetic force and lowers the cyclotron frequency. If the center of cyclotron gyration is displaced from the center of the radial electric field, there is an additional magnetron gyration. That motion amounts to a slow circular drift of the center of cyclotron gyration along an isopotential contour about the center of the radial electric field. The magnetron gyration is driven by the magnetic force that results from the radial ion velocity induced by the force from the radial electric field.

\section{Multipole Ion Guide}

The critical feature of an electrodynamic ion guide like the multipole is that the electrode geometry results in an $\mathrm{rf}$ electric field whose magnitude increases with radial distance from the central axis (where the field is zero). An ion displaced from the axis is subject to a force from the electric field that causes a radial oscillation at the rf drive frequency; the so-called wiggling or micromotion. A consequence of the radial field inhomogeneity is that the average force during that oscillation is directed toward the axis and increases with distance from the axis. The force is often conceptualized as the result of a displacement of the ion from the minimum of a static radial potential well known as the effective potential or pseudopotential. If the radial restoring force is sufficient to contain the ion, it causes an additional radial oscillation that is slower and larger in amplitude than the micromotion and variously referred to as macro-, guiding center, or secular motion.

\section{Magnetomultipole}

Trajectory simulations reveal that the radial ion trajectory of a non-resonant ion in the magnetomultipole is a superposition of three types of motion: a circular gyration near the cyclotron frequency, a much slower circular drift around the multipole axis, and a low amplitude oscillation near the rf drive frequency (Figure 2). These motions are analogous to the cyclotron gyration, magnetron gyration, and micromotion described above; however, the sense of the magnetron motion is typically opposite to that in the Penning/ICR trap and the direction and magnitude of the cyclotron frequency shift is determined by the phase and frequency of the rf field. If the $\mathrm{rf}$ frequency is near or equal to $n$ times the effective cyclotron frequency, the electric field additionally affects the cyclotron amplitude in a manner analogous to off-resonance or resonant excitation of cyclotron motion in a Penning/ICR trap $[24,26]$. A resonant ion is continuously accelerated by the electric field of the magnetomultipole and possibly ejected; whereas an ion near resonance exhibits a periodic variation of the cyclotron radius as the ion is alternately accelerated and decelerated by the electric field. The amplitude of this periodic variation or "beat" motion varies inversely with the difference between the cyclotron and resonant frequency and may be sufficient to cause ejection of the ion. 

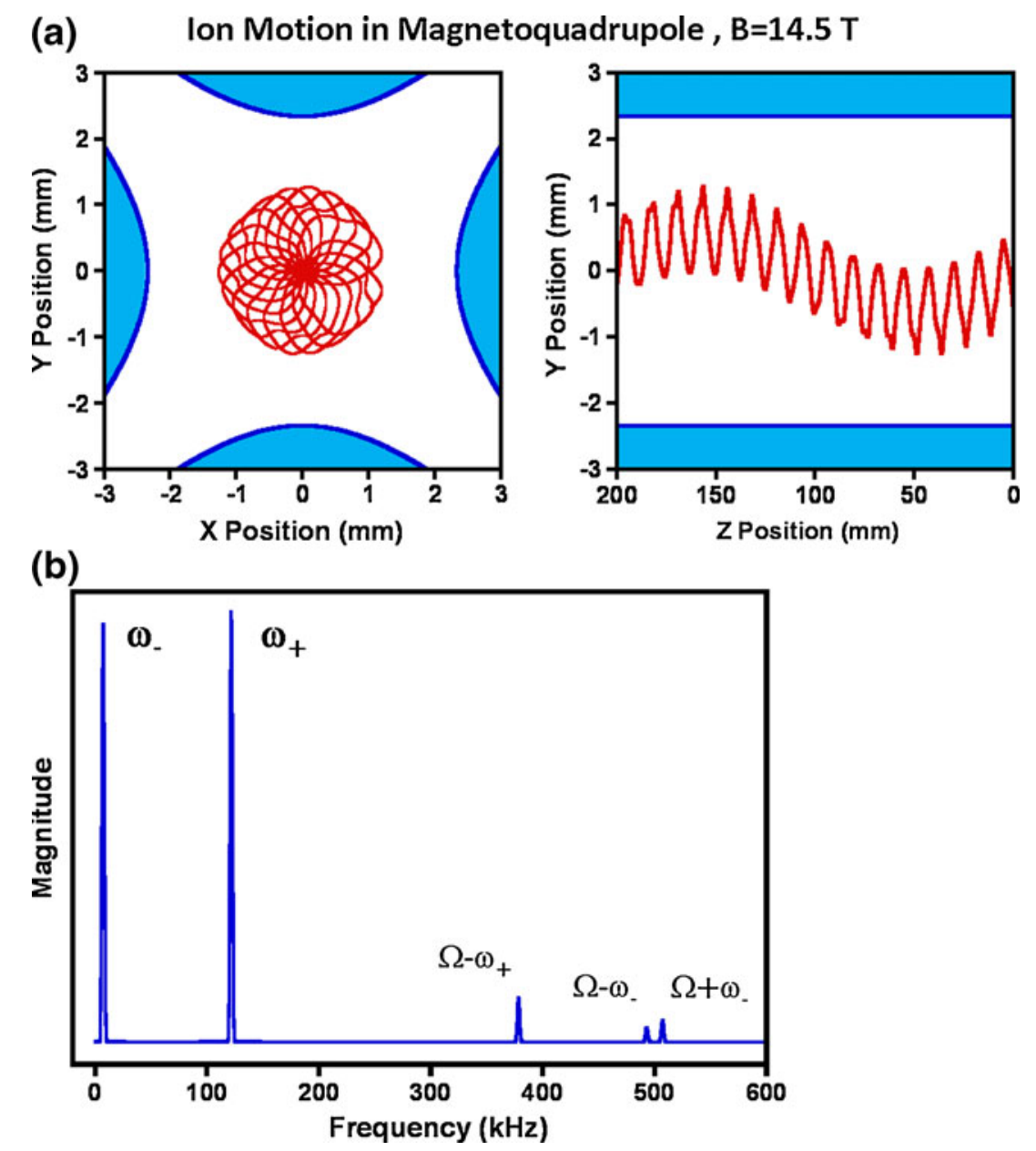

Figure 2. (a) Final trajectory in a magnetoquadrupole for an ion of $\mathrm{m} / \mathrm{z} 2000$ with initial average radial kinetic energy of $0.5 \mathrm{eV}$ and initial axial kinetic energy of $25 \mathrm{eV}$. Magnetic field is $14.5 \mathrm{~T}$; rf potential is $90 \mathrm{~V}_{p-\mathrm{p}}$ at $500 \mathrm{kHz}$. (b) Fourier transform spectrum of the final time-domain radial trajectory component in (a)

\section{Magnetic Field Gradient}

Ions that traverse the magnetic field gradient associated with external source FT-ICR MS exhibit characteristic motion that progresses from that of the multipole ion guide to that of the magnetomultipole (Figure 1). The transition is most easily visualized for an ion originating on the magnetomultipole axis with initial radial kinetic energy insufficient to escape the effective multipole potential well. In the absence of a magnetic field, the ion oscillates at the secular frequency in a plane containing the multipole axis. As the ion encounters a continuously increasing magnetic field, the $\mathbf{v} \times \mathbf{B}$ force causes the plane of oscillation to rotate around the axis, and the radial trajectory assumes the shape of loops radiating from the axis (the so-called rose curve of analytic geometry). With increasing magnetic field the cyclotron mode eventually dominates the radial motion and the ion assumes a nearly circular orbit tangent to the magnetomultipole axis. If the ion ultimately encounters a magnetic field at which the ion cyclotron frequency equals the rf drive frequency divided by the multipole order, the trajectory assumes the familiar spiral path resulting from continuous radial acceleration by the resonant electric field (Figure 1). This transient cyclotron resonance may result in radial ejection of the ion if the electric field encountered at the ion's initial cyclotron radius is sufficiently large.

The magnetic field gradient includes a radial component that varies with distance from the axis and is small relative to the axial field component within the inscribed radius of the typical multipole. For example, the maximum radial magnetic field encountered within the inscribed radius of the multipoles simulated in this work is 0.054 Tesla at a position where the axial field is 8.6 Tesla. During resonance, the ion motion is dominated by the force associated with the much larger axial field and the effect of the radial field component may be reasonably ignored. The principal impact of the radial magnetic field is manifest prior to resonance as ions pass through the gradient and $\mathbf{v} \times \mathbf{B}$ interaction with the radial field causes axial-to-radial kinetic energy transfer that increases the ion cyclotron radius $[19,20]$. The increase is insufficient to cause radial ejection; however, the effect significantly lowers the initial radial kinetic energy an ion 
may possess upon entering the magnetomultipole without later being subject to ejection by transient cyclotron resonance.

\section{Resonant Acceleration of Radial Ion Motion}

The resonant response of an ion to an electric field within the magnetomultipole can be determined by methods previously employed for analyzing excitation modes within the FT-ICR trap [27]. The time averaged power absorption from the electric field $\langle P\rangle$, is determined by integrating the product of the ion velocity with the parallel component of the force due to the electric field

$$
\langle P\rangle=\frac{1}{T} \int_{0}^{T} q \boldsymbol{E} \cdot \boldsymbol{v} d t
$$

Change in motional amplitude resulting from power absorption is assumed to be slow relative to the motional frequency, so that $\boldsymbol{v}$ may be approximated as the superposition of velocity vectors associated with simple circular motion in each of the radial modes. Although the micromotion in the magnetomultipole is not circular, the amplitude is typically small relative to that of magnetron and cyclotron modes and may be ignored at this level of approximation. The magnetron and cyclotron modes are represented as circular orbits with corresponding frequencies $\omega_{-}$and $\omega_{+}$, and radii $\rho_{-}$and $\rho_{+}$to yield

$$
\begin{aligned}
\boldsymbol{v}_{x y}=\{ & -\rho_{-} \omega_{-} \sin \left(\omega_{-} t\right)-\rho_{+} \omega_{+} \sin \left(\omega_{+} t\right), \rho_{-} \omega_{-} \cos \left(\omega_{-} t\right) \\
& \left.+\rho_{+} \omega_{+} \cos \left(\omega_{+} t\right)\right\}
\end{aligned}
$$

in which the initial phase of both modes has been set to 0 radians to simplify subsequent expressions. Substitution of Equations 2 and 5 into Equation 4 yields the following expressions for time-averaged power absorption in a quadrupole, hexapole, and octopole:

$$
\begin{aligned}
\langle P\rangle_{\text {Quad }}= & \frac{1}{T} \int_{0}^{T} \frac{q V_{p-\mathrm{p}} \cos (\Omega t+\phi)}{r_{0}^{2}}\left[\rho_{-}^{2} \omega_{-} \sin \left(2 \omega_{-} t\right)\right. \\
& \left.+\rho_{-} \rho_{+}\left(\omega_{-}+\omega_{+}\right) \sin \left(\left(\omega_{-}+\omega_{+}\right) t\right)+\rho_{+}^{2} \omega_{+} \sin \left(2 \omega_{+} t\right)\right]
\end{aligned}
$$

$$
\begin{aligned}
\langle P\rangle_{H e x}= & \frac{1}{T} \int_{0}^{T} \frac{3 q V_{p-p} \cos (\Omega t+\phi)}{2 r_{0}^{2}}\left[\rho_{-}^{3} \omega_{-} \sin \left(3 \omega_{-} t\right)\right. \\
& +\rho_{-}^{2} \rho_{+}\left(2 \omega_{-}+\omega_{+}\right) \sin \left(\left(2 \omega_{-}+\omega_{+}\right) t\right)+\rho_{-} \rho_{+}^{2}\left(\omega_{-}\right. \\
& \left.\left.+2 \omega_{+}\right) \sin \left(\left(\omega_{-}+2 \omega_{+}\right) t\right)+\rho_{+}^{3} \omega_{+} \sin \left(3 \omega_{+} t\right)\right]
\end{aligned}
$$

$$
\begin{aligned}
\langle P\rangle_{O c t}= & \frac{1}{T} \int_{0}^{T} \frac{2 q V_{p-p} \cos (\Omega t+\phi)}{r_{0}^{2}}\left[p_{-}^{4} \omega_{-} \sin \left(4 \omega_{-} t\right)\right. \\
& +\rho_{-}^{3} \rho_{+}\left(3 \omega_{-}+\omega_{+}\right) \sin \left(\left(3 \omega_{-}+\omega_{+}\right) t\right) \\
& +3 \rho_{-}^{2} \rho_{+}^{2}\left(\omega_{-}+\omega_{+}\right) \sin \left(2\left(\omega_{-}+\omega_{+}\right) t\right) \\
& +\rho_{-} \rho_{+}^{3}\left(\omega_{-}+3 \omega_{+}\right) \sin \left(\left(\omega_{-}+3 \omega_{+}\right) t\right) \\
& \left.+\rho_{+}^{4} \omega_{+} \sin \left(4 \omega_{+} t\right)\right]
\end{aligned}
$$

The cosine product integrals are maximized if the arguments have the same frequency and a phase difference of $\pi / 2$. That condition corresponds to resonant excitation and occurs if $\phi=$ $\pi / 2$ and the multipole rf frequency is equal to $n \omega_{-}, n \omega_{+}$, or any of several combination frequencies corresponding to $k \omega_{-}+(n-k) \omega_{+}$ in which $k=1 \cdots n-1$. Excitation at $n \omega_{-}$or $n \omega_{+}$, results in continuous power absorption; whereas excitation at any of the combination frequencies causes finite energy absorption and periodic interconversion of energy between the magnetron and cyclotron modes. Interconversion caused by quadrupolar and octopolar excitation has been previously described and exploited to make high precision mass measurements of short-lived isotopes [28-30], and to manipulate ions in the FT-ICR [31-34].

The ion magnetron and cyclotron frequencies change with the local magnetic field as ions traverse the magnetic field gradient spanned by the multipole used for external source FTICR MS. The range of the magnetron frequency variation remains small enough that the resonance at $n \omega_{\text {- }}$ is not encountered for the typical case that $\Omega \gg>n \omega_{-}$; however, the range of the cyclotron frequency variation is large enough that each of the other resonances is encountered for ions below an associated mass-to-charge ratio determined by the frequency of the resonance. Excitation of the combination resonances may cause some periodic variation in radial displacement of the ion, but is unlikely to result in radial ejection for initial magnetron and cyclotron radii consistent with introduction of ions near the multipole axis. The $n \omega_{+}$resonance causes a continuous increase in radial displacement that may result in ejection of the ion and is therefore the subject of further analysis.

\section{Excitation of the $n \omega_{+}$Resonance}

The change in radius during resonant excitation can be determined by assuming that the time-averaged power absorbed by the ion is manifest as a time rate of change in kinetic energy of the resonant mode [27], which leads to the following expression for cyclotron mode excitation

$$
\langle P\rangle=\frac{d}{d t}\left(\frac{m \omega_{+}^{2} \rho_{+}^{2}}{2}\right)=m \omega_{+}^{2} \rho_{+} \dot{\rho}_{+}
$$

Equation 7 can be solved for the time rate of change of the cyclotron radius $\rho_{+}(t)$, during resonant excitation. For excitation of the $n \omega_{+}$resonance we set $\Omega=n \omega_{+}$in Equations 6a-c, integrate over one period of $n \omega_{+}$, substitute 
the result into Equation 7 and solve the differential equations to obtain

$$
\begin{gathered}
\rho_{+}(t)_{\text {quad }}=\rho_{0} \exp \left(\frac{C}{2} t\right) \\
\rho_{+}(t)_{\text {hex }}=\left(\frac{1}{\rho_{0}}-\frac{3 C}{4}\right)^{-1} \\
\rho_{+}(t)_{\text {oct }}=\left(\frac{1}{\rho_{0}^{2}}-2 C t\right)^{-1 / 2}
\end{gathered}
$$

in which $\rho_{0}$ is the initial cyclotron radius and

$$
C=\frac{q V_{p-\mathrm{p}}}{m \omega_{+} r_{0}^{n}}
$$

Equation $8 \mathrm{a}$ was previously derived (with an appropriate geometry factor) to describe azimuthal quadrupolar excitation at $2 \omega_{+}$in a cubic FT-ICR trap [27] and expresses an exponential increase in ion cyclotron radius with time on resonance. Equations $8 \mathrm{~b}$ and $8 \mathrm{c}$ indicate that ion cyclotron radius increases asymptotically with time on resonance for the hexapole and octopole.

\section{Effective Cyclotron Frequency}

Equations 7-9 describe resonant excitation of cyclotron motion caused by the component of the electric field vector that is parallel to the cyclotron velocity vector. We now consider the effect of the electric field component perpendicular to the cyclotron velocity vector, because the resulting force on the ion combines with the perpendicular force from the magnetic field to shift the effective cyclotron frequency and determine the $\mathrm{m} / \mathrm{z}$ subject to resonant excitation.

\section{Motional Averaging}

Ion motion in the magnetomultipole may be analyzed in the context of a motionally averaged electric field in an approach similar to that described for the multipole ion guide above. The component of the average field that is perpendicular to the ion cyclotron velocity is

$$
\left\langle E_{c}^{\perp}\right\rangle=\frac{1}{T} \int_{0}^{T} \boldsymbol{E}^{\cdot} \widehat{\boldsymbol{v}}_{c}^{\perp} d t
$$

in which $\widehat{\boldsymbol{v}}_{c}^{\perp}$ is the unit vector perpendicular to the of the ion cyclotron velocity vector

$$
\widehat{\boldsymbol{v}}_{c}^{\perp}=\left\{-\cos \left(\omega_{+} t\right),-\sin \left(\omega_{+} t\right)\right\}
$$

Here again the initial phase has been set to 0 radians to simplify subsequent expressions. Substitution of Equations 2 and 11 into Equation 10 yields the following expressions for the perpendicular component of the time-averaged electric field in the quadrupole, hexapole, and octopole:

$$
\begin{gathered}
\left\langle E_{c}^{\perp}\right\rangle_{\text {Quad }}=\frac{1}{T} \int_{0}^{T}-\frac{V_{p-\mathrm{p}} \cos (\Omega t+\phi)}{r_{0}^{2}} \\
{\left[\rho_{-} \cos \left(\left(\omega_{-}+\omega_{+}\right) t\right)+\rho_{+} \cos \left(2 \omega_{+} t\right)\right]} \\
\left\langle E_{c}^{\perp}\right\rangle_{\text {Hex }}=\frac{1}{T} \int_{0}^{T}-\frac{3 V_{p-\mathrm{p}} \cos (\Omega t+\phi)}{2 r_{0}^{3}} \\
{\left[\rho_{-}^{2} \cos \left(\left(2 \omega_{-}+\omega_{+}\right) t\right)+2 \rho_{-} \rho_{+} \cos \left(\left(\omega_{-}+2 \omega_{+}\right) t\right)\right.} \\
\left.\quad+\rho_{+}^{2} \cos \left(3 \omega_{+} t\right)\right] \\
\left\langle E_{c}^{\perp}\right\rangle_{\text {Oct }}=\frac{1}{T} \int_{0}^{T}-\frac{2 V_{p-\mathrm{p}} \cos (\Omega t+\phi)}{r_{0}^{4}} \quad(12 \mathrm{c}) \\
{\left[\rho_{-}^{3} \cos \left(\left(3 \omega_{-}+\omega_{+}\right) t\right)+3 \rho_{-}^{2} \rho_{+} \cos \left(2\left(\omega_{-}+\omega_{+}\right) t\right)\right.} \\
\left.+3 \rho_{-} \rho_{+}^{2} \cos \left(\left(\omega_{-}+3 \omega_{+}\right) t\right)+\rho_{+}^{3} \cos \left(4 \omega_{+} t\right)\right]
\end{gathered}
$$

For excitation of the $n \omega_{+}$resonance we set $\Omega=n \omega_{+}$and integrate over one period of $\omega_{+}$. The result may be generalized according to multipole order to obtain

$$
\left\langle E_{c}^{\perp}\right\rangle=-\frac{n V_{p-\mathrm{p}}}{4 r_{0}}\left(\frac{\rho_{+}}{r_{0}}\right)^{n-1} \cos \phi
$$

An important implication of the $\cos \phi$ dependence in Equation 13 is that a range of $\mathrm{m} / \mathrm{z}$ values is subject to the $n \omega_{+}$resonance for a particular value of $\Omega$.

\section{Resonant $m / z$ Range}

Equation 1 may be recast for cyclotron motion in a plane perpendicular to $\boldsymbol{B}=(0,0, B)$ as

$$
m \omega_{+}^{2} \rho_{+}=q\left\langle E_{c}^{\perp}\right\rangle+q B \omega_{+} \rho_{+}
$$

Equation 14 may be solved for the resonant case of $\omega_{+}=$ $\Omega / n$ after substitution of Equation 13 to obtain

$$
m=-\frac{n^{3} q V_{p-\mathrm{p}}}{4 \Omega^{2} r_{0}^{2}}\left(\frac{\rho_{+}}{r_{0}}\right)^{n-2} \cos \phi+\frac{n q B}{\Omega}
$$


An ion not initially in resonance exhibits a phase $\phi$ that varies continuously such that $\cos \phi$ ranges from -1 to 1 . Resonance may thereby obtain within an $\mathrm{m} / \mathrm{z}$ range centered about $n q B / \Omega$ and with boundaries determined by substituting \pm 1 for $\cos \phi$ in Equation 15:

$$
m_{\text {range }}=\frac{n q B}{\Omega} \pm \frac{n^{3} q V_{p-p}}{4 \Omega^{2} r_{0}^{2}}\left(\frac{\rho_{+}}{r_{0}}\right)^{n-2}
$$

Ions traversing a magnetic field gradient, 0 to $B_{0}$, in the magnetomultipole are subject to an effective $\mathrm{m} / \mathrm{z}$ resonance band that extends from 0 to the upper band limit defined in Equation 16. The upper limit establishes an $\mathrm{m} / \mathrm{z}$ ejection threshold below which resonant ejection of ions may occur

$$
m_{\text {thresh }}=\frac{n q B_{0}}{\Omega}+\frac{n^{3} q V_{p-p}}{4 \Omega^{2} r_{0}^{2}}\left(\frac{\rho_{+}}{r_{0}}\right)^{n-2}
$$

The threshold increases linearly with $V_{p \text {-p }}$ for all multipole geometries; however, sensitivity to $\rho_{+}$varies exponentially with $n$ for $n>2$ because of nonlinear variation of $\left\langle E_{c}{ }^{\perp}\right\rangle$ with $\rho_{+}$in higher order multipoles. The effect of $\rho_{+}$can be understood by considering the $\rho_{+}$dependence of each of three force terms in Equation 14. The centrifugal and magnetic forces vary linearly with $\rho_{+}$and are unaffected by multipole geometry; however, the electric force is proportional to $\rho_{+}{ }^{n-1}$ as shown in Equation 13. The electric force varies linearly with $\rho_{+}$in the quadrupole and the balance of forces shown in Equation 14 is therefore unaffected by $\rho_{+}$; however, in higher order multipoles $\omega_{+}$must increase with $\rho_{+}$to maintain the balance of forces.

\section{Results and Discussion}

\section{Resonant Power Absorption at $n \omega_{+}$}

Simion was used to model variation of cyclotron radius with time for $\Omega=n \omega_{+}$and the results were compared to the analytical approximations of Equations $8 \mathrm{a}-\mathrm{c}$ for each of the three magnetomultipoles in a homogeneous magnetic field of $14.5 \mathrm{~T}$ (Figure 3). The initial ion radial kinetic energy was $0.1 \mathrm{eV}(\phi=0)$ and the initial axial kinetic energy was $0 \mathrm{eV}$ so that ions remain in the magnetomultipole long enough to be ejected (i.e., until $\rho_{+}=r_{0}$ ). rf Parameters were selected to yield similar effective potential well depth and ion transfer characteristics for each multipole in the absence of a magnetic field: $90 V_{p \text {-p }}$ at $0.5 \mathrm{MHz}$ for the magnetoquadrupole; $122 V_{p-p}$ at $1.0 \mathrm{MHz}$ for the magnetohexapole, and 150 $V_{p \text {-p }}$ at $1.5 \mathrm{MHz}$ for the magneto-octopole. Equations $8 \mathrm{a}-\mathrm{c}$ closely approximate the variation of cyclotron radius with time, as evidenced by $\mathrm{R}^{2}$ values of $0.991,0.995$, and 0.997 for the magnetoquadrupole, -hexapole, and -octopole.
Simulated data for the magnetoquadrupole exhibit small periodic deviations from Equation $8 \mathrm{a}$ caused by radial acceleration that is fast compared to $\omega_{+}$; however, all of the correlations approach unity as $V_{p \text {-p }}$ and initial radius are reduced to achieve the slow change in radius relative to cyclotron frequency assumed in the derivation of Equations $8 \mathrm{a}-\mathrm{c}$.

\section{Resonant $\mathrm{m} / \mathrm{z}$ Range}

\section{Magnetoquadrupole}

The $m / z$ range over which resonant ejection at $n \omega_{+}$occurs in the magnetoquadrupole is determined by specifying $n=2$ in Equation 16 to obtain

$$
m_{\text {quad }}=\frac{2 q B}{\Omega} \pm \frac{2 q V_{p-\mathrm{p}}}{\Omega^{2} r_{0}^{2}}
$$

Trajectory simulations were used to determine the variation of resonant $\mathrm{m} / \mathrm{z}$ ejection range with $V_{p \text {-p }}$ for $\Omega=$ $0.5 \mathrm{MHz}$ in a homogeneous magnetic field of $14.5 \mathrm{~T}$. Equation 18 very closely approximates the simulated ejection band, as evidenced by $\mathrm{R}^{2}=1.000$ for both the upper and lower boundaries of the band (Figure 4).

\section{Magnetohexapole and-Octopole}

Presentation of ejection band data for the magnetohexapole and -octopole is complicated by the $\rho_{+}$dependence for $n>2$ in Equation 16:

$$
m_{\text {hex }}=\frac{3 q B}{\Omega} \pm \frac{27 q V_{p-\mathrm{p}} \rho_{+}}{4 \Omega^{2} r_{0}^{3}}
$$

$$
m_{o c t}=\frac{4 q B}{\Omega} \pm \frac{8 q V_{p-\mathrm{p}} \rho_{+}^{2}}{\Omega^{2} r_{0}^{4}}
$$

Quantitative interpretation of $\rho_{+}$is complicated because it increases continuously for an ion with $\mathrm{m} / \mathrm{z}$ within the resonance band; whereas an ion with $\mathrm{m} / \mathrm{z}$ just outside the band undergoes off-resonance excitation with periodic variation of $\rho_{+}$. The effective band limits are therefore determined by the maximum $\rho_{+}$achieved by the ion prior to encountering resonance, which is in turn determined by initial ion kinetic energy and relative phase angle, $\phi$. Although a thorough analysis of the $n>2$ resonance band is beyond the scope of this work, it is sufficient to note the influence of multipole parameters and that the width of the resonant $\mathrm{m} / \mathrm{z}$ band increases with initial ion kinetic energy. 
(a)

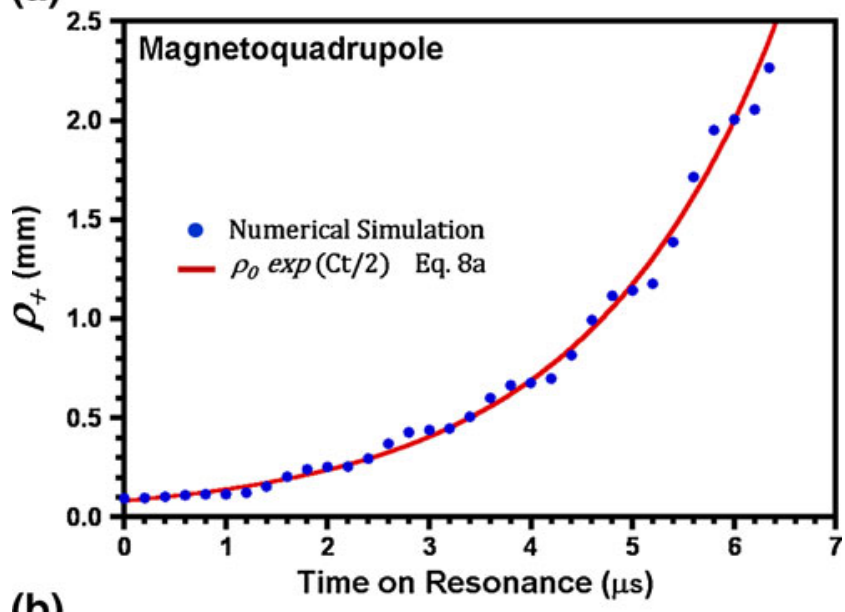

(b)

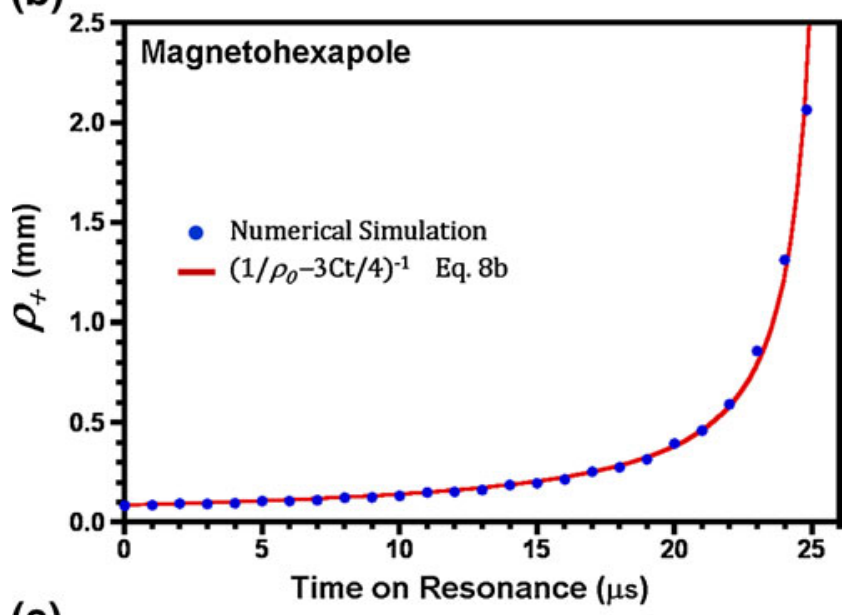

(c)

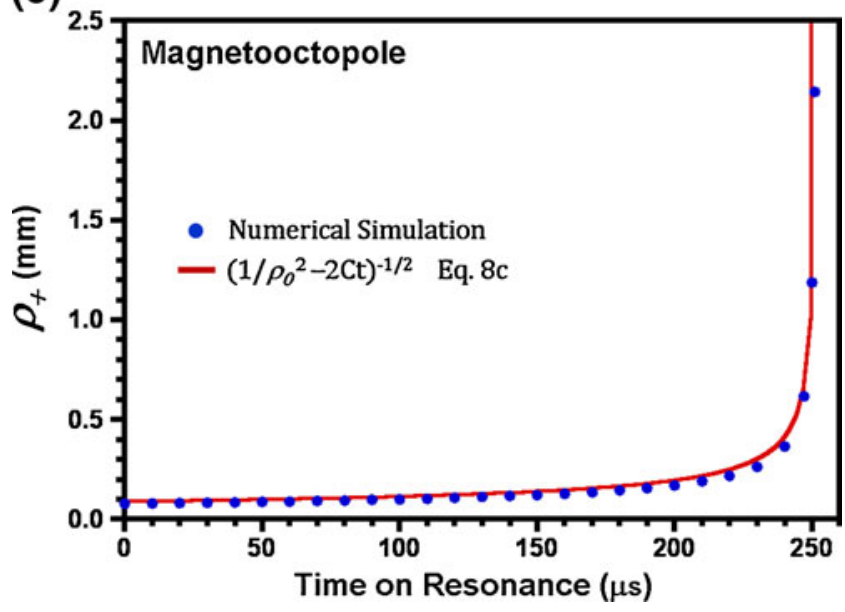

Figure 3. Ion cyclotron radius versus time on resonance for (a) magnetoquadrupole, (b) magnetohexapole, and (c) magneto-octopole

\section{Ion Transfer Efficiency}

Variation of ion transfer efficiency with $\mathrm{m} / \mathrm{z}$ and initial radial kinetic energy was determined for each of the three multi- pole geometries (Figure 5). Otherwise identical simulations were run with and without a magnetic field gradient to explicitly demonstrate the effect of the added field. The principal effect of the magnetic field gradient is significantly attenuated transfer efficiency for ions of lower $\mathrm{m} / \mathrm{z}$, attributable to resonance at $n \omega_{+}$

\section{Magnetoquadrupole}

Transfer attenuation is sharp and essentially complete below what amounts to a low $m / z$ cutoff for the magnetoquadrupole (Figure 5a, top). The cutoff varies linearly with $V_{p \text {-p }}$ as predicted by Equation 17 (Figure 6a), and is independent of initial ion radial kinetic energy (Figure 5b, top). Radial kinetic energy independence is consistent with the absence of $\rho_{+}$in Equation 17 for $n=2$, and is responsible for the sharp cutoff.

\section{Magnetohexapole and-Octopole}

The magnetic field does not impose a complete transmission cut-off for the magnetohexapole and -octopole (Figure 5a, middle and bottom), but instead significantly attenuates transmission below an $\mathrm{m} / \mathrm{z}$ threshold that depends on initial radial kinetic energy (Figure 5b, middle and bottom). The threshold extends from a local minimum in transmission at $m=n q B / \Omega$ to a $V_{p \text {-p }}$-dependent higher $m / z$ corresponding to the onset of attenuation (Figure 6b). That behavior is qualitatively consistent with the appearance of $\rho_{+}$in Equation 17 for $n>2$. The minimum results from prolonged opportunity for resonance as the magnetic field reached by the ions has leveled off at $B_{0}$ in the region preceding the exit end of the multipole; however, transmission does not drop to zero at the minimum or lower $\mathrm{m} / \mathrm{z}$ because of the low radial

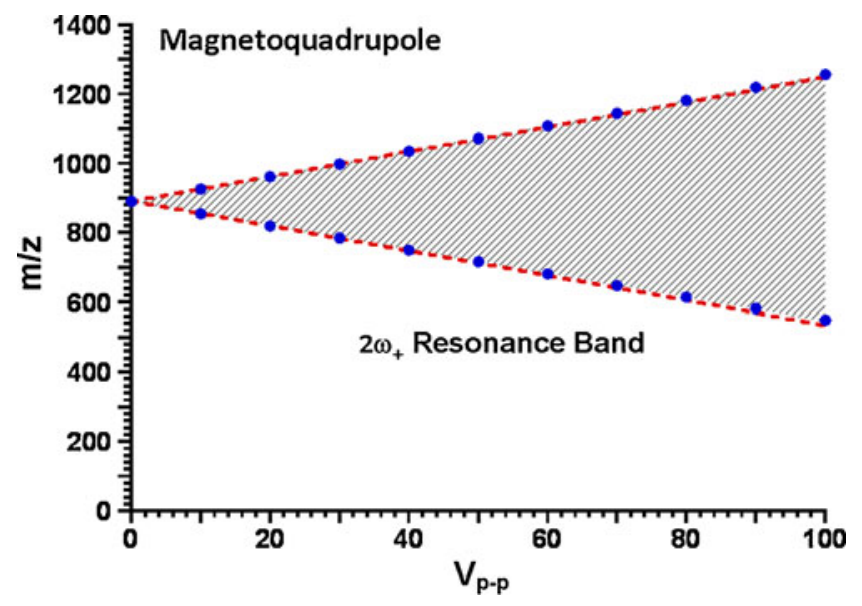

Figure 4. The $2 \omega_{+}$resonance band for a magnetoquadrupole, determined from numerical trajectory simulations $(\bullet)$ and Equation 17 (- - -) in a homogeneous static magnetic field of 14.5 T. All ions with $\mathrm{m} / \mathrm{z}$ within the hatched band are ejected from the multipole 
electric field at low radii in higher order multipoles. The multipole radial field varies as $\left(\rho+/ r_{0}\right)^{n-1}$, and for similar effective pseudopotential well depth, higher order multipoles have correspondingly lower radial field at a given radius, and lower average radial field over the inscribed radius. Only ions with sufficient radial kinetic energy and corre-

(a)

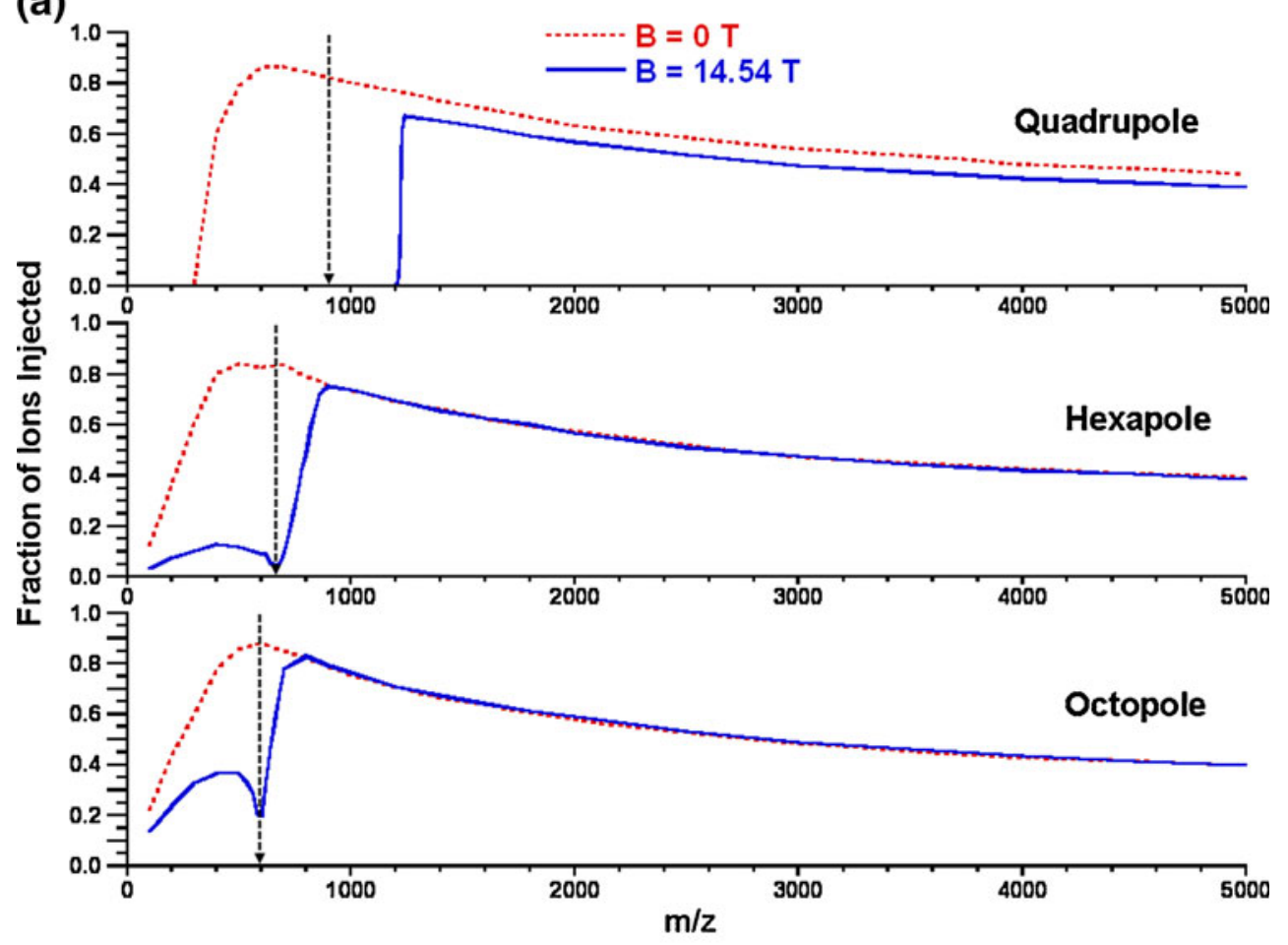

(b)

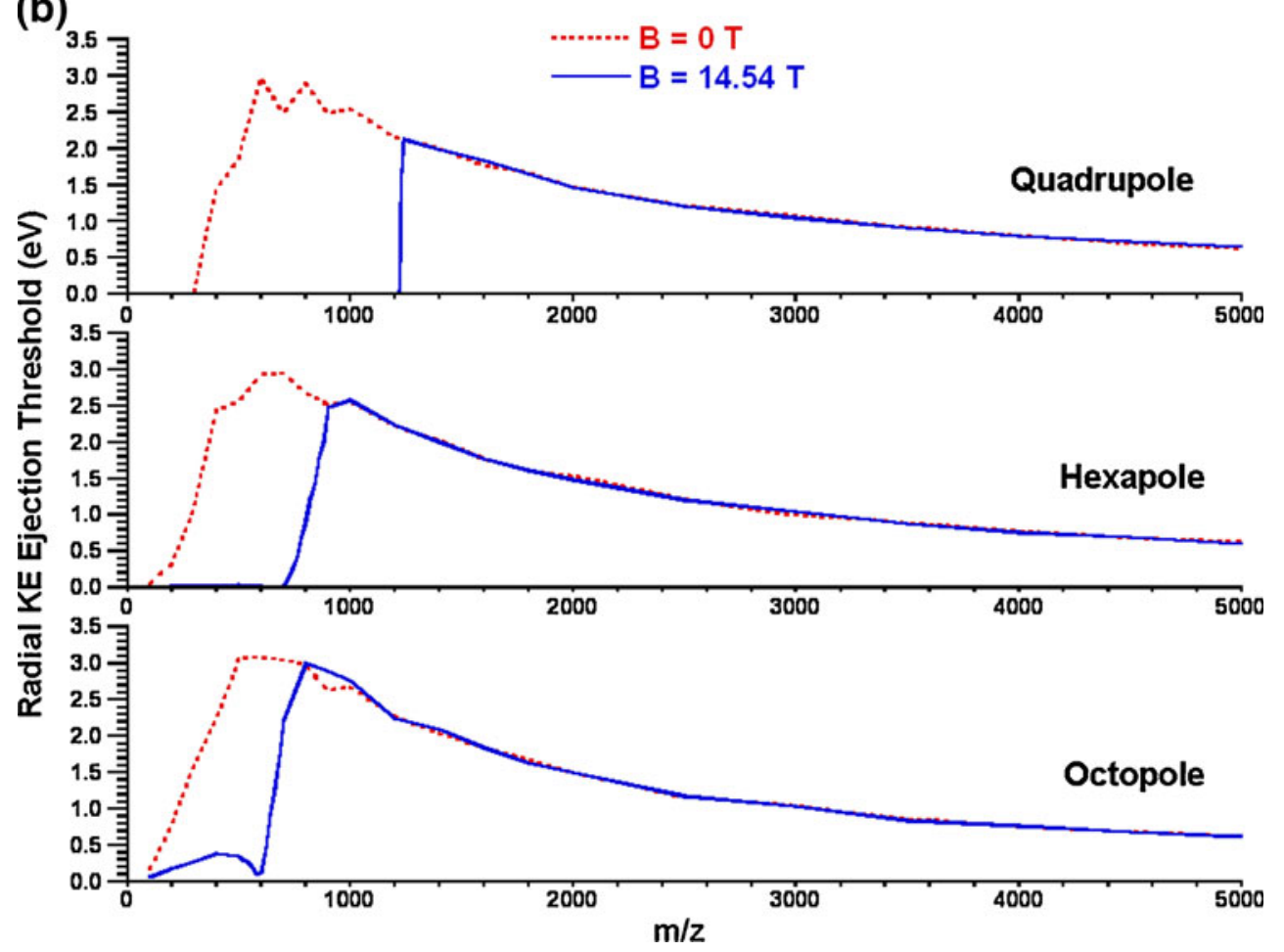

Figure 5. (a) lon injection efficiency, and (b) radial kinetic energy ejection threshold, vs. $m / z$ with (-), and without $(---)$ a $0-$ 14.5 T axial magnetic field gradient. The rf frequency and amplitude for each multipole were selected to provide similar injection efficiency profiles in the absence of a magnetic field (quadrupole $=0.5 \mathrm{MHz}, 90 V_{p-p}$; hexapole=1.0 MHz, $122 V_{p \text {-p }}$; octopole $=$ $\left.1.5 \mathrm{MHz}, 150 V_{p-p}\right)$. Note that these conditions also result in similar ejection threshold curves in (b) 
sponding radial amplitude absorb enough energy to be ejected during the transient resonance period. Ion radial kinetic energy increases during traversal of the magnetic field gradient prior to resonance, so the initial ion radial kinetic energy at the entrance of the magnetomultipole must be significantly lower than this local threshold to avoid ejection.

\section{Radial Kinetic Energy Ejection Thresholds}

The ejection thresholds presented in Figure $5 \mathrm{~b}$ indicate the maximum initial ion radial kinetic energy at the entrance of the magnetomultipole without subsequent ejection caused by transient cyclotron resonance. The maximum corresponds to ions with initial cyclotron motion that is in phase with the multipole $\mathrm{rf}$ drive (i.e., $\pi / 2$ radians ahead of the electric field vector [26]) at resonance. Ions that are out of phase with the rf drive decelerate prior to achieving resonant acceleration, and may therefore avoid ejection with an initial radial kinetic energy that is greater than that in Figure $5 \mathrm{~b}$.

The radial kinetic energy ejection threshold is further affected by any azimuthal ion velocity component at the magnetomultipole entrance (i.e., a component perpendicular to the plane containing the multipole axis). An azimuthal velocity component introduces additional $\boldsymbol{v} \times \boldsymbol{B}$ interaction with the radial component of the magnetic field gradient and thereby changes the extent of axial-to-radial kinetic energy conversion prior to resonance. Significant azimuthal velocity results only for ions not well focused on the axis upon entrance to the magnetomultipole and is not included in the present work.

\section{Conclusions}

Analytic and numerical modeling reveal that ions traveling through a multipole ion guide operated in a static magnetic field are susceptible to radial ejection caused by transient cyclotron resonance that occurs when the local effective cyclotron frequency equals the multipole rf drive frequency divided by the multipole order. The resonance extends over an $\mathrm{m} / \mathrm{z}$ range that varies with $\mathrm{rf}$ amplitude and, if the magnetic field incorporates a large gradient such as that associated with external source FT-ICR MS, spans the low $\mathrm{m} / \mathrm{z}$ transmission range of the multipole up to an $\mathrm{m} / \mathrm{z}$ threshold that varies with multipole parameters. The present theoretical treatment of transient cyclotron resonance in a multipole ion guide includes a quantitative description of resonant cyclotron acceleration as a function of multipole parameters and initial ion radial kinetic energy. Additional relations predict the $\mathrm{m} / \mathrm{z}$ range susceptible to resonance.

For multipole parameters that yield comparable pseudopotential well depth, susceptibility to resonant ejection as determined by initial ion radial kinetic energy decreases with multipole order; quadrupole $>>$ hexapole $>$ octopole. The $\mathrm{m} / \mathrm{z}$ range subject to resonance is primarily determined by the ratio of multipole order to multipole drive frequency, and
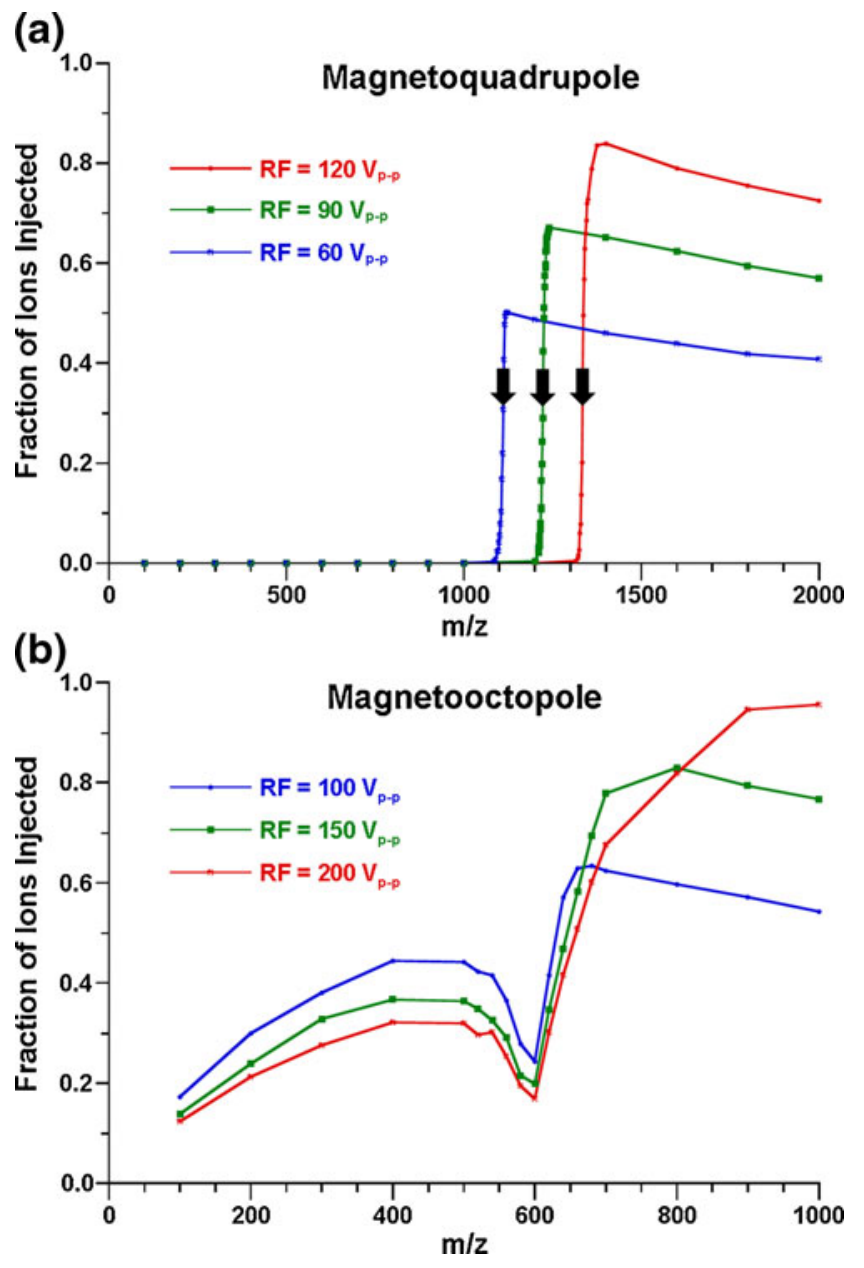

Figure 6. Variation of ion injection efficiency with $\mathrm{m} / \mathrm{z}$ for three different rf amplitudes in (a) magnetoquadrupole, and (b) magneto-octopole, with 0-14.5 $\mathrm{T}$ axial magnetic field gradient. The $\mathrm{m} / \mathrm{z}$ ejection threshold predicted by Equation 17 is indicated by the arrows superposed on the transmission curves in (a)

to lesser extent the drive amplitude. For comparable drive frequency the resonant $\mathrm{m} / \mathrm{z}$ range increases with multipole order; quadrupole $<$ hexapole $<$ octopole.

The evolution of FT-ICR MS has been largely driven by the implementation of ever higher magnetic fields because of the resulting improvement in instrument figures of merit. Unfortunately, the $\mathrm{m} / \mathrm{z}$ range over which transient cyclotron resonance occurs increases with magnetic field and is therefore more likely to overlap the desired mass spectral range. One way to reduce the $\mathrm{m} / \mathrm{z}$ range susceptible to resonance is to increase the multipole rf frequency; however, this approach may require impractically high $\mathrm{rf}$ power to drive the reactive load presented by the multipole. We have recently modeled the alternative of shortening the multipole so that it does not extend the full distance from the source to the ICR cell, but instead terminates in a lower field region of the magnetic field gradient [35]. The reduced maximum magnetic field experienced by ions while in the magneto- 
multipole was shown to result in a proportionate reduction in the $m / z$ threshold below which transient resonance occurs. Future work will establish practical limits for multipole length reduction as determined by the influence on final ion properties (e.g., kinetic energy and radial distribution), and further characterize the exchange of axial and radial ion kinetic energy that occurs for non-resonant ions in the magnetomultipole [19, 20].

\section{Acknowledgments}

The authors acknowledge support for this by NSF Division of Materials Research through DMR-0654118 and the State of Florida.

\section{References}

1. Marshall, A.G.: Milestones in fourier transform ion cyclotron resonance mass spectrometry technique development. Int. J. Mass Spectrom. 200, 331-356 (2000)

2. Chen, F.F.: Introduction to plasma physics. Plenum Press, New York (1974)

3. McIver Jr., R.T., Hunter, R.L., Bowers, W.D.: Coupling a quadrupole mass spectrometer and a Fourier transform mass spectrometer. Int. J. Mass Spectrom. Ion Processes 64, 67-77 (1985)

4. McIver, R.T.: Trajectory calculations for axial injection of ions into a magnetic field: overcoming the magnetic mirror effect with an $\mathrm{rf}$ quadrupole lens. Int. J. Mass Spectrom. Ion Processes 98, 35-50 (1990)

5. Gerlich, D. Inhomogeneous rf fields: a versatile tool for the study of processes with slow ions. In: Advances in Chemical Physics; CheukYiu Ng, Michael Baer, Ilya Prigogine, Stuart A. Rice, Eds., John Wiley \& Sons, New York, 1992; pp 1-176

6. Hägg, C., Szabo, I.: New ion-optical devices utilizing oscillatory electric fields. II. Stability of ion motion in a two-dimensional hexapole field. Int. J. Mass Spectrom. Ion Processes 73, 237-275 (1986)

7. Hägg, C., Szabo, I.: New ion-optical devices utilizing oscillatory electric fields. III. Stability of ion motion in a two-dimensional octopole field. Int. J. Mass Spectrom. Ion Processes 73, 277-294 (1986)

8. Hägg, C., Szabo, I.: New ion-optical devices utilizing oscillatory electric fields. IV. Computer simulations of the transport of an ion beam through an ideal quadrupole, hexapole, and octopole operating in the rf-only mode. Int. J. Mass Spectrom. Ion Processes 73, 295-312 (1986)

9. Huang, Y., Guan, S., Kim, H.S., Marshall, A.G.: Ion transport through a strong magnetic field gradient by r.f.-only octupole ion guides. Int. J. Mass Spectrom. Ion Processes 152, 121-133 (1996)

10. Li, G.-Z., Werth, G.: The combined trap and some possible applications. Phys. Scr. 46, 587-592 (1992)

11. O, C.-S., Schuessler, H.A.: Confinement of ions created externally in a quadrupole ion trap operated in the combined Penning and radiofrequency mode. J. Appl. Phys. 52, 2601-2607 (1981)

12. Yan, M., Luo, X., Zhu, X.: Potential usage of the magnetron-motionfree mode of one ion confined in a combined trap. Appl. Phys. B Lasers Opt. 67, 235-239 (1998)

13. Nakamura, T., Ohtani, S., Wada, M., Okada, K., Katayama, I., Schuessler, H.A.: Ion dynamics and oscillation frequencies in a linear combined trap. J. Appl. Phys. 89, 2922-2931 (2001)

14. Schuessler, H.A., Benck, E.C., Lassen, J.: A linear combined trap for on-line spectroscopy. Hyperfine Interact. 81, 263-273 (1993)

15. Bate, D.J., Dholakia, K., Thompson, R.C., Wilson, D.C.: Ion oscillation frequencies in a combined trap. J. Mod. Opt. 39, 305-316 (1992)

16. Huang, Y., Li, G.-Z., Guan, S., Marshall, A.G.: A combined linear ion trap for mass spectrometry. J. Am. Soc. Mass Spectrom. 8, 962-969 (1997)

17. Beu, S.C., Hendrickson, C.L., Marshall, A.G. SIMION modeling of rfonly multipole ion guides for injection of externally generated ions in high field FT-ICR MS Proceedings of the 52nd ASMS Annual Conferenc on Mass Spectrometry and Allied Topics, Nashville, TN, 23-27 May, 2004, WPH132

18. Beu, S.C., Hendrickson, C.L., Marshall, A.G. SIMION modeling of ion transfer in rf-only multipole ion guides immersed in strong magnetic field gradients Proceedings of the 55th ASMS Annual Conferenc on Mass Spectrometry and Allied Topics, Indianapolis, IN, 3-7 June, 2007, MPD070

19. Beu, S.C., Hendrickson, C.L., Marshall, A.G. Changes in ion kinetic energy distribution during transfer through a multipole ion guide in a strong magnetic field gradient Proceedings of the 56th Annual Conferenc on Mass Spectrometry and Allied Topics, Denver, CO, 1-5 June, 2008, TPA010.

20. Beu, S.C., Hendrickson, C.L., Marshall, A.G. Exchange of axial and radial kinetic energy during ion transfer through multipole ion guides in a strong magnetic field gradient Proceedings of the 57th Annual Conferenc on Mass Spectrometry and Allied Topics, Philadelphia, PA, 31 May - 4 June, 2009, TPK279

21. Zekavat, B., Solouki, T. Injection of externally-generated low mass ions into high magnetic field Q/FT-ICR instruments: simion simulations and experimental observations. Annual Conferenc on Mass Spectrometry and Allied Topics, Salt Lake City, UT, 23-27 May, 2010, MP 655

22. Friedman, M.H., Yergey, A.L., Campana, J.E.: Fundamentals of ion motion in electric radio-frequency multipole fields. J. Phys. E Sci. Instrum. 15, 53-61 (1982)

23. Mickens, R.E.: An introduction to nonlinear oscillations. Cambridge University Press, Cambridge [Eng.]; New York (1981)

24. Marshall, A.G., Hendrickson, C.L., Jackson, G.S.: Fourier transform ion cyclotron resonance mass spectrometry: a primer. Mass Spectrom. Rev. 17, 1-35 (1998)

25. Brown, L.S., Gabrielse, G.: Geonium theory: physics of a single electron or ion in a penning trap. Rev. Mod. Phys. 58, 233-311 (1986)

26. Wang, M., Marshall, A.G.: Laboratory-frame and rotating-frame ion trajectories in ion cyclotron resonance mass spectrometry. Int. J. Mass Spectrom. Ion Processes 100, 323-346 (1990)

27. Schweikhard, L., Marshall, A.G.: Excitation modes for Fourier transform-ion cyclotron resonance mass spectrometry. J. Am. Soc. Mass Spectrom. 4, 433-452 (1993)

28. Becker, S., Bollen, G., Kern, F., Kluge, H.J., Moore, R.B., Savard, G., Schweikhard, L., Stolzenberg, H.: Mass measurements of very high accuracy by time-of-flight ion cyclotron resonance of ions injected into a penning trap. Int. J. Mass Spectrom. Ion Processes 99, 53-77 (1990)

29. Ringle, R., Bollen, G., Schury, P., Schwarz, S., Sun, T.: Octupolar excitation of ion motion in a Penning trap - a study performed at LEBIT. Int. J. Mass Spectrom. 262, 33-44 (2007)

30. Eliseev, S., Block, M., Chaudhuri, A., Herfurth, F., Kluge, H.J., Martin, A., Rauth, C., Vorobjev, G.: Octupolar excitation of ions stored in a Penning trap mass spectrometer - a study performed at SHIPTRAP. Int. J. Mass Spectrom. 262, 45-50 (2007)

31. Guan, S., Marshall, A.G.: Bloch equations applied to ion cyclotron resonance spectroscopy: broadband interconversion between magnetron and cyclotron motion for ion axialization. J. Chem. Phys. 98, 4486-4493 (1993)

32. Guan, S., Wahl, M.C., Marshall, A.G.: Broadband axialization in an ion cyclotron resonance ion trap. J. Chem. Phys. 100, 6137-6140 (1994)

33. Schweikhard, L., Guan, S., Marshall, A.G.: Quadrupolar excitation and collisional cooling for axialization and high pressure trapping of ions in Fourier transform ion cyclotron resonance mass spectrometry. Int. J. Mass Spectrom. Ion Processes 120, 71-83 (1992)

34. Breitenfeldt, M., Ziegler, F., Herlert, A., Marx, G., Schweikhard, L.: Simultaneous monitoring of the radial modes of the ion motion and their manipulation in Penning traps by FT-ICR mass spectrometry. Int. J. Mass Spectrom. 263, 94-100 (2007)

35. Beu, S.C., Hendrickson, C.L., Marshall, A.G. Design consideration for external ion injection FT-ICR MS at 21 Tesla. Proeedings of the. 58th ASMS. Annual Conference on Mass Spectrometry and Allied Topics, Salt Lake City, UT, 23-27 May, 2010, MP 651 